\title{
Training in neurology: lessons learnt
}

\author{
Samuel Shribman, ${ }^{\oplus}$ Sian K Alexander, ${ }^{\oplus 2}$ Angelika Zarkali, ${ }^{3}$ \\ Thomas T Warner, ${ }^{1}$ Anthony C Pereira, ${ }^{4}$ Tom A T Hughes, ${ }^{5}$ \\ Catherine J Mummery ${ }^{3}$
}

- Additional material is published online only. To view please visit the journal online (http://dx.doi.org/10.1136/ practneurol-2018-002129).

${ }^{1}$ Reta Lila Weston Institute of Neurological Studies, UCL Queen Square Institute of Neurology, London, UK ${ }^{2}$ Department of Neurology, Addenbrooke's Hospital, Cambridge, UK

${ }^{3}$ Dementia Research Centre, National Hospital for Neurology and Neurosurgery, London, UK ${ }^{4}$ Department of Neurology, St George's Hospital, London, UK ${ }^{5}$ Department of Neurology, University Hospital of Wales, Cardiff, UK

\section{Correspondence to}

Dr Catherine J Mummery, Dementia Research Centre, National Hospital for Neurology and Neurosurgery, London WC1N 3BG, UK; cath mummery@nhs.net

Accepted 31 March 2019 Published Online First 13 May 2019

\section{SLinked}

- http://dx.doi.org/10.1136/ practneurol-2019-002313

\section{Check for updates}

(c) Author(s) (or their employer(s)) 2019. No commercial re-use. See rights and permissions. Published by BMJ.

To cite: Shribman $S$,

Alexander SK, Zarkali A, et al. Pract Neurol 2019;19:431-

437.

\begin{abstract}
There is no consensus on how to structure and deliver neurology training. The General Medical Council's annual National Training Survey indicates that the quality of UK neurology training is very variable, but does not explain this variation. We used the survey data to identify the four highest and lowest performing sites for neurology training across the UK. We conducted semistructured interviews with groups of local trainees and, separately, local trainers in an exploratory qualitative study, and identified common themes across a range of aspects of neurology training. Here we present our findings, share case studies from top-performing sites and make recommendations on how best to train a neurologist.
\end{abstract}

\section{INTRODUCTION}

Specialist training in neurology involves a combination of apprenticeship, teaching and self-directed learning, in parallel with clinical practice. Neurologists have to appreciate a diverse range of symptoms, signs and diseases, and be able to make complex diagnoses and decisions on lifechanging and high-risk interventions in emergency and outpatient settings.

UK neurology trainees rotate over 5 years between a small number of university-based teaching hospitals and district general hospitals within a deanery (figure 1). There are 15 deaneries, some of which are further divided into rotations; trainees rarely change deanery after starting their neurology training. ${ }^{1} \mathrm{~A}$ national curriculum provides an outline of the skills, knowledge and experience expected of a trained neurologist; a professional portfolio with annual reviews is used to monitor progress. ${ }^{2}$ However, there is no consensus or guidance on how to structure and deliver neurology training at a local level.

Anecdotally, trainees report significant variability in their experience of training within and outside their deanery; open-access data from the General Medical Council (GMC) National Training Survey support this. ${ }^{3}$ Annual completion of the survey is mandatory for all UK trainees and the responses are used to derive scores for a range of training-related indicators. An overall satisfaction indicator is derived by combining responses to five general questions about training (box 1).

The GMC website shows data on neurology training at 33 of the 66 sites (hospitals) where neurologists were being trained in 2018 (figure 1); the remaining 33 sites had responses from fewer than three trainees and are excluded to maintain the anonymity of respondents. Overall satisfaction scores ranged from $42 \%$ to 98\% (figure 2). According to the GMC's analysis, one site performed significantly above and three sites performed significantly below (defined by non-overlapping 95\% CIs) the national average across all non-neurological specialties.

Faced with evidence of variability in training delivery and trainee satisfaction, the key question is what determines the quality of training at a given site. In order to identify successful models for how to train a neurologist at a local level, we used the Training Survey data to identify the highest and lowest performing sites, and then used semistructured interviews in an exploratory qualitative study to probe the methods and practices of training in these sites.

\section{METHODS}

We selected four of the highest and four of the lowest performing sites for the interviews. These were chosen from the highest or lowest six sites based on mean overall satisfaction scores over the last 5 years; our final choice of sites ensured adequate geographic variation and inclusion of both medium and large sites. Small sites, with fewer than three trainees, were already excluded. We chose not to disclose 


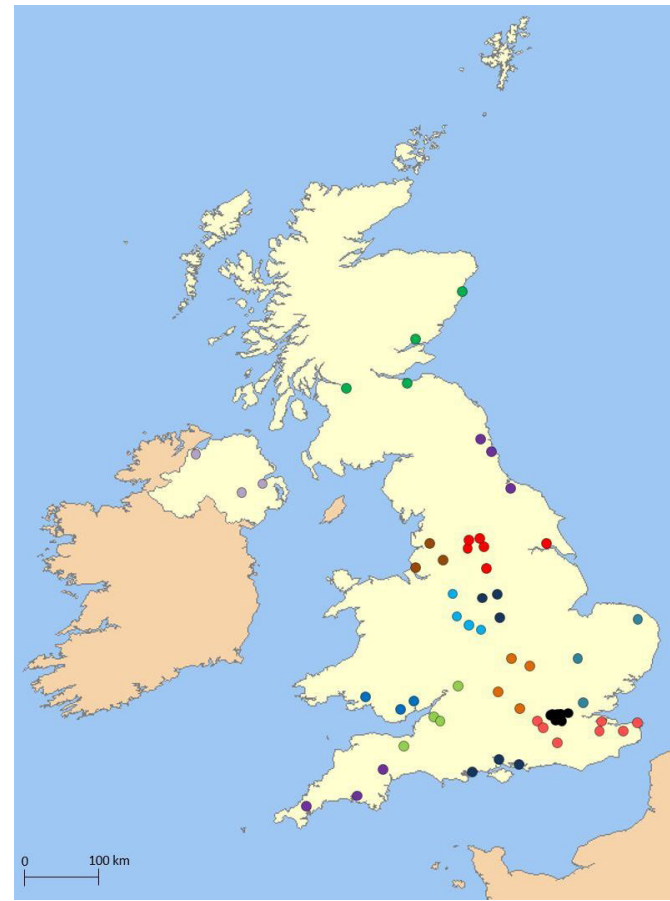

Figure 1 A map showing neurology training sites across the UK. The 66 sites identified in the General Medical Council's (GMC) National Training Survey in 2018 are shown and colour-coded according to deanery. A table with the name of each site, rotation and deanery is provided in the online supplementary material (map adapted by ChrisO from https:// commons.wikimedia.org/wiki/File:Uk_outline_map.png under the terms of the GNU Free Documentation License, V.1.2).

the names of the sites visited to maintain interviewees' anonymity.

We conducted semistructured interviews with trainees, and then separately and individually with local trainer(s). We interviewed trainees in groups of three to eight, with no trainer(s) present. We interviewed one to three trainers at each site except one, where none were available. All interviews were conducted by one of three trainees using standardised questions

Box 1 Questions from the General Medical Council's (GMC) National Training Survey that are used to derive overall satisfaction scores

Please rate the quality of clinical supervision in this post.

- How would you rate the quality of experience in this post?

- How would you describe this post to a friend who was thinking of applying for it?

- To what extent do you agree with the following statement? 'This post will be useful for my future career.'

- Please rate the quality of teaching (informal and bedside teaching as well as formal and organised sessions) in this post.

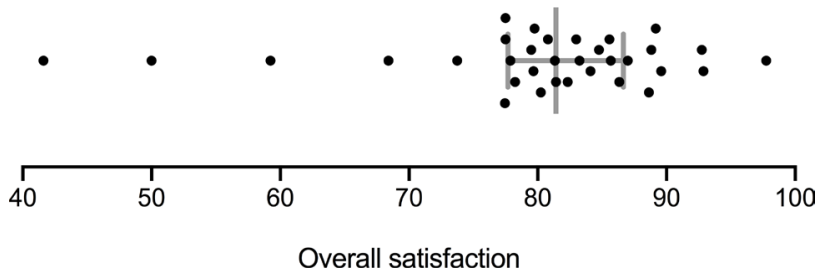

Figure 2 General Medical Council's (GMC) National Training Survey scores for overall satisfaction in neurology training at 33 sites across the UK in 2018. The error bars indicate the median and inter-quartile range.

(see online supplementary material) over 45-60 min, and either audio recorded or transcribed directly by the interviewer. A minority of data were collected by telephone interview and email.

The interviews focused on training at the local level. We asked trainees 26 prespecified questions (and trainers 20) about the structure, effectiveness and safety of training at that site. We started with openended questions, such as 'How would you describe your department?' and 'What is the best/worst thing about training at this trust?' followed by more specific questions, such as 'To what extent do consultants supervise you in the following situations?' and 'How is local teaching delivered?'

We undertook a thematic analysis using a combination of deductive and inductive reasoning after completing all the interviews. The three interviewers initially read each transcript independently to compare responses to individual questions and to identify emerging themes over three meetings. Given the relatively small number of interviews, we defined key themes as those occurring in at least two of four high-performing sites and in no low-performing sites, or in at least three of four high-performing sites and one low-performing site (and vice versa).

We made additional analyses on the National Training Survey data. We compared overall satisfaction scores with data on clinic attendance using Spearman's correlation coefficient. We also compared scores for overall satisfaction between those deaneries with trainees given unsatisfactory outcomes in their annual review, with those who had only satisfactory outcomes, using an unpaired t-test. The data were provided by the Joint Royal Colleges of Physicians Training Board. We compared scores for overall satisfaction in 2018 with that in each of the 5 preceding years using the Wilcoxon signed-rank test. We performed statistical analyses using GraphPad Prism V.8.

\section{CLINICAL SUPERVISION}

The most discernible differences between high and low-performing sites related to the form and extent of clinical supervision for inpatient neurology. Trainees at most top-performing sites would meet the supervising neurologist face to face to discuss the ward referrals that they had reviewed or the telephone advice they 
had given that day. They would then make a shared decision about which inpatients required subsequent review by the supervisor in person. At most of these sites, a specific time to meet during the working day was decided in advance; this allowed trainees to manage their time more effectively. Statements from trainees at top-performing sites included, 'every case gets discussed, even if it is a very brief summary of what we've done, we run it by the boss' and 'even if I don't need to call them there and then, I would always say these are the people I've seen, this is what I've done, are you happy?'

By contrast, at most low-performing sites there was a problem with access to the supervising neurologist. Clinical supervision depended on the trainee contacting their supervisor via telephone, when she/ he felt it was necessary. Statements from trainees at low-performing sites included, 'with most [supervisors] you call them as and when you need them' and that reviewing patients was 'generally never volunteered'. This was associated with lack of supervision across inpatient activities, including telephone triage, ward reviews and intensive care referrals. It also contributed to a marked discrepancy between the trainers' expectations of when trainees should contact the supervising neurologist and when trainees did contact the supervising neurologist.

Of concern, trainees at several of these sites reported difficulty arranging a review by the supervising neurologist on the same or following day and, in some situations, at all. Statements from trainees at low-performing sites included '[supervisors] very rarely question or examine patients' and 'when they do go and see a patient it tends to be very superficial; I'm getting an opinion from [them] but [they] are completely relying on what I've said'.

We identified several working patterns that facilitated supervision:

- Having a weekly, as opposed to a daily, rota for the supervising neurologist.

- Reducing or cancelling outpatient clinics during their on-call week.

- Dividing responsibility for supervising different aspects of inpatient care (see Case study 1).

The two most cited barriers to effective supervision were: the supervising neurologist being off-site due to other clinical responsibilities; and a lack of structure, creating uncertainty as to when, where and how the trainee should meet their supervisor. Indicative trainee comments were 'sometimes they are not physically here' and 'even the really good [supervisors] don't really see referrals; maybe once or twice a week they'll come to see a patient you're worried about'; 'it's particularly hard for people starting their training here'.

There were similar differences in supervision for training in outpatient neurology. Trainees at most high-performing sites tended to have more supervision, particularly when reviewing new cases, whereas trainees at several of the low-performing sites frequently ran outpatient clinics without any supervision. This was a particular problem for rapid access clinics at several sites. By contrast, trainees and trainers at top-performing sites reported it was particularly rewarding to follow-up their own patients.

\section{COMPETENCY-BASED TRAINING}

Training at top-performing sites was tailored to the experience and competency of neurology trainees. Junior trainees were expected to discuss all inpatient and outpatient cases with the supervising neurologist whereas more senior trainees were encouraged to practise more independently, contacting the supervising neurologist when necessary. There were also differences in the clinical roles and approaches to induction. Examples of good practice included junior trainees shadowing senior trainees when on call for several weeks or months at the start of their training, being allocated specific training rotations, or seeing outpatient cases selected by their supervisor in advance (see Case study 2).

In several sites a senior trainee, usually in their final year, was appointed to a role that included elements of leadership and management; where successful, this role bridged between trainees and the lead trainer. Responsibilities included the allocation of training rotations, rota design and, in some situations, clinical support for more junior trainees. This approach was viewed as a valuable learning experience for the senior trainee, and good preparation for working life after training, provided their job plans recognised their additional responsibilities.

A unique problem at top-performing sites was the risk of oversupervision and 'spoon-feeding'. This was acknowledged by trainers and trainees alike and highlights the universal importance of tailored, competency-based training.

\section{CLINIC ATTENDANCE}

Neurology is predominantly an outpatient specialty and neurology trainees are recommended to work in a minimum of two clinics per week. Using GMC National Training Survey data on clinic attendance, we identified large variations between sites, which could imply significantly different overall clinical experience between trainees. Median clinic attendance across 33 sites ranged from one to four clinics per week. It was fewer than two clinics per week at $12 \%$ of sites, and three or more clinics per week at $21 \%$ of sites (figure 3).

There was no correlation between clinic attendance and GMC National Training Survey scores $(\mathrm{p}=0.089)$. However, trainees at high and low-performing sites noted that staffing levels influenced their ability to attend clinics: unfilled posts, often for more junior doctors, meant trainees being diverted away from clinics where they were supernumerary in order to cover referrals, ambulatory care units and inpatient wards. One trainee said, "Our clinic exposure is remarkably poor; between 


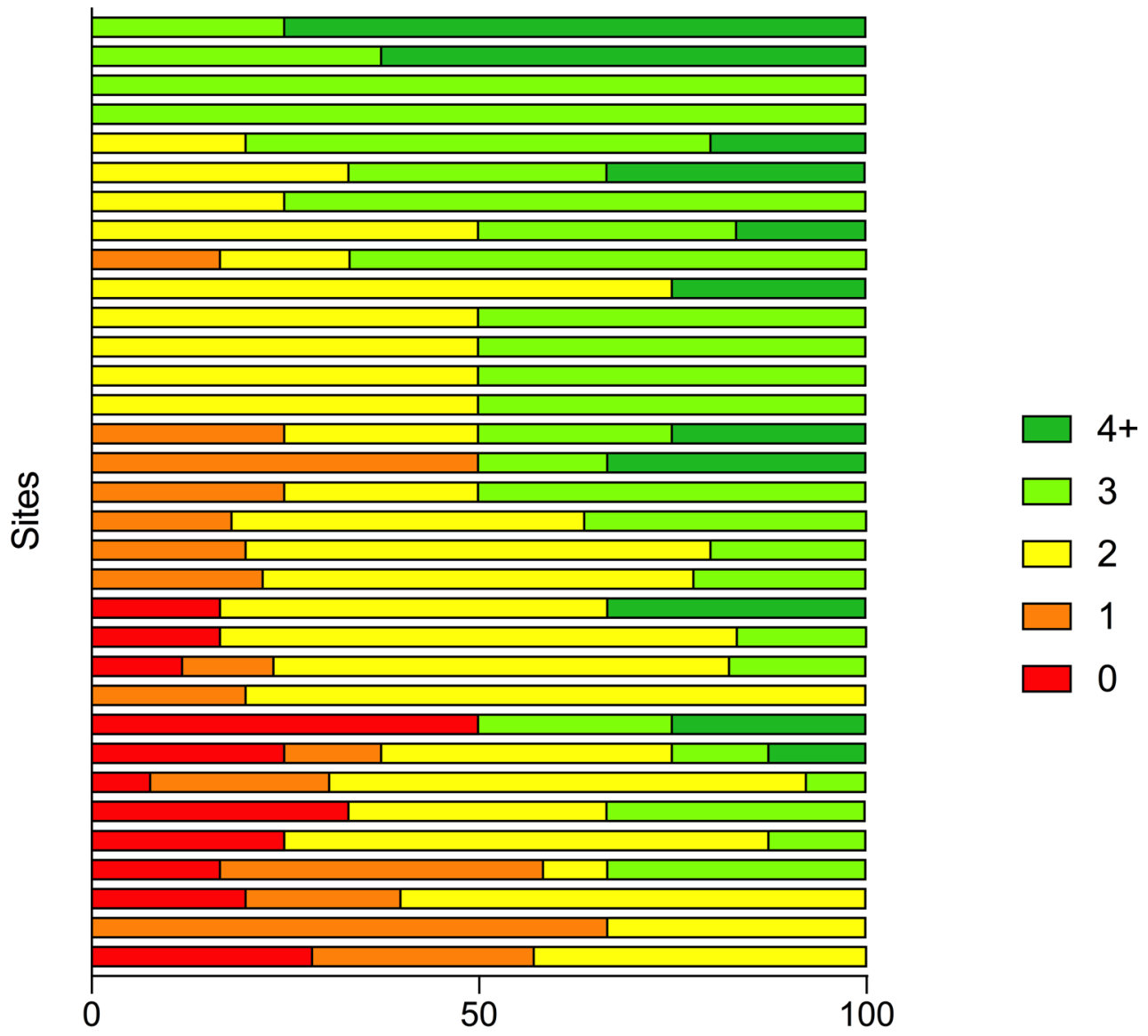

$\%$ of trainees

Figure 3 General Medical Council's (GMC) National Training Survey results for average clinic attendance per week at 33 sites across the UK in 2018. The percentage of neurology trainees who attend a given number of clinics per week at a given site is represented in each horizontal bar.

four [trainees] we are expected to be in three clinics per week but because the workload is so intense [we] hardly manage to fit in one."

We did not specifically examine the balance between general and subspecialty neurology and whether trainees were supernumerary or had their own patient list in outpatient clinics. We will explore these issues through additional questions specific to neurology trainees in future GMC National Training Surveys.

\section{ROLES AND RESPONSIBILITIES}

Trainers at several top-performing sites clearly defined the roles and responsibilities of the supervising consultant and trainee. The supervising consultant 'own[s] the episode of care and has a responsibility to support the trainee to ensure that they are able to administer their responsibilities effectively'; 'the trainee is there to be trained, which involves a mixture of observation and practice but always on a supernumerary basis.' A trainee in a low-performing unit explained, "As a registrar, you are very often doing the job of the consultant, registrar, senior house officer and secretary."
Protected time for trainees to complete administrative responsibilities (reviewing clinic letters, outpatient investigations, and so on) was incorporated to the working week at one top-performing site. This was highly valued by the trainees and further highlights the importance of effective job planning. In contrast, a trainee at a low-performing site said, "We always end up doing admin rather than sitting in on clinic."

Inadequate junior doctor staffing was a recurring theme at low-performing sites with neurology trainees performing tasks on a daily basis such as rewriting drug charts, prescribing fluids, venepuncture, cannulation and catheterisation. One trainee said, "We're chronically understaffed without any [junior doctor] cover for a lot of the time, sometimes for weeks." By contrast, no high-performing site reported inadequate junior doctor staffing. Trainees at some low-performing sites reported being asked by their supervising consultant to arrange outpatient investigations for patients who were not under their care. This incurred the risk of failing to identify contraindications or omitting key clinical information on request forms, was unlikely to enhance training and is an unsafe practice. 
High and low-performing sites had a similar number of educational meetings for review of training portfolio progress; all trainees met their educational supervisors two to three times each year. Thus, the number of educational meetings does not necessarily relate to the quality of training at a local level despite these being mandatory for satisfactory outcomes at annual appraisals. We did not, however, explore the content of these educational meetings during our interviews.

\section{SUBSPECIALTY EXPOSURE}

There was a range of subspecialty training opportunities available at most sites. Trainees at some low-performing sites reported difficulty achieving adequate subspecialty exposure and cited the main reason as being competing clinical priorities due to inadequate junior doctor staffing. Most high-performing sites used elective rotations or sessions for educational time within the working week to ensure adequate exposure; trainees greatly valued this (see Case study 3). A further, simple initiative at a top-performing site was a trainee-compiled list of valuable learning opportunities around their site (eg, autonomic testing, neuro-ophthalmology clinics, radiology reporting sessions, multidisciplinary team meetings, theatre lists) including timings and contact details for the relevant specialists. This made it easy for trainees quickly to identify and attend suitable opportunities according to their schedules.

All neurology trainees are expected to become familiar with cerebrovascular disease: although it features in the curriculum, dedicated time on a stroke unit or completion of a stroke medicine curriculum is not mandatory for neurology trainees in the UK. While our interviews did not specifically examine access to stroke training, we are informally aware of significantly varied exposure to stroke across the UK. The GMC National Training Survey data showed that $61 \%$ of neurology trainees are involved in the delivery of acute stroke services in the UK in 2018.

\section{LOCAL TEACHING}

There was a large variation in local teaching programmes. All trainees valued teaching howsoever it was delivered. High-performing sites had more frequent and varied teaching programmes with multiple sessions each week, including small-group seminars or bedside teaching, as well as wider departmental teaching. One site had daily teaching in various formats (see Case study 4). By contrast, most teaching at some low-performing sites was provided through departmental meetings that were routinely used for clinical governance or business purposes. One trainee said, "There is a teaching rota but it only happens once per month."

Several other themes emerged at top-performing sites. First, teaching followed a fixed timetable without variation in timing or location. Second, a neurologist separate to the training lead was responsible for the departmental teaching programme. At one site this neurologist used the specialty curriculum and trainee feedback to guide topics for internal and external speakers and optimise curriculum coverage. Third, a subset of neurologists would rotate chairing departmental meetings. Fourth, trainees presenting cases would be expected to discuss the case with a supervising neurologist before their presentation.

At all sites it was acknowledged that the on-call trainee might be unable to attend local or regional teaching opportunities, but that this effect would be shared across all trainees during the course of the training programme.

\section{CULTURE}

There were also differences in the culture and ethos within departments. At top-performing sites there was a feeling that both trainees and trainers prioritised training over service delivery, that trainees knew what was expected of them and that trainers knew what to expect of trainees. Other specific examples included shared coffee or tea breaks after a busy ward round or clinic, and trainees feeling comfortable to knock on the door of their supervisor for advice during clinics. Trainees at both high and low-performing sites valued shared trainee office space, which engendered a supportive environment with peer and near-peer supervision.

Trainers and trainees identified positive aspects of training predominantly at a single centre, where the strengths, weakness and overall trajectory of trainees are better understood and training relationships develop over several years: one trainee explained that it was 'impossible to fly under the radar' training there. Where trainees were based at a single site throughout training, there was a predictably shorter commute time (under $35 \mathrm{~min}$ on average), reflecting trainees' decisions to live closer to work. By contrast, trainees at several low-performing sites raised issues of long commutes or needing to relocate. The average commute time was over an hour, and at one site, most trainees had needed temporarily to relocate mid-way through training to be closer to work. Trainees perceived these issues as particularly onerous. They may increase the risk of burnout, which is a growing cross-speciality concern in the UK and an acknowledged problem for neurologists in other countries. ${ }^{4}$

Finally, the culture within departments was also reflected by social activities outside work. Trainees at high-performing sites reported meeting colleagues outside work on at least a monthly, as opposed to annual, basis. Unlike low-performing sites, this was usually in the context of the wider clinical team, including both supervisors and more junior colleagues. In isolation, these activities have the potential to feel artificial and onerous but they appeared to enhance the feeling of belonging, collegiality and informality for 
Box 2 Recommendations, with specific examples, for how to train a neurologist

Clinical supervision should involve regular face-to-face discussions and sequential consultation by the trainee and supervising neurologist in selected cases.

- Trainees meet the supervising neurologist to discuss inpatient referrals and review selected cases at a preallocated time during the working day.

- Supervising neurologists follow a weekly, as opposed to a daily, rota.

- Supervising neurologists cancel clinics, or convert them to rapid access clinics, for on-call weeks.

- Supervising neurologists are available to review any new outpatient referral, including those seen in rapid access clinics, if needed.

The level of supervision should be matched to experience and capability of the trainee.

- Junior trainees shadow senior trainees on call at the start of their training.

- Junior trainees start on rotations with the most supervision.

- Junior trainees discuss all new and follow-up cases in clinics.

- Supervisors identify suitable patients for trainees to see prior to clinic.

- Senior trainees are encouraged to see cases independently or have their own clinic lists.

- A senior trainee has clinical and managerial responsibilities incorporated into his/her job plan.

The burden of administrative and non-training service work should be minimised.

- Dedicated sessions are scheduled for reviewing clinic letters and investigation results.

- Trainees do not arrange investigations/appointments for outpatients that they do not know.

- Routine and simple clinical tasks should be performed by appropriately trained nurses, clinical assistants or more junior members of the team.

Local challenges in providing subspecialty training should be identified and addressed.

- Protected sessions for subspecialty activities are scheduled in the weekly timetable.

- Elective rotations are included in the training programme.

- Lists of local subspecialist training opportunities are generated by, or for, trainees.

- Gaps in trainees' subspecialty experience are identified before allocating training rotations.

- Departmental teaching content is guided by the neurology curriculum and feedback.

Departments should promote a culture of enquiry, learning and mutual support.

- Local teaching has a fixed, weekly timetable including practical and seminar-based sessions.

\section{Box 2 Continued}

- Departmental teaching is chaired by one of several neurologists with an interest in teaching.

- Trainees share a communal office space.

- Regular social activities for the wider department are encouraged.

trainees and trainers alike. Understanding the wider lives of our colleagues should lead to greater appreciation for them and create an environment in which most of us would prefer to work and train.

\section{CONCLUSIONS}

The optimal training outcome for both current and future delivery of safe, timely and excellent neurological patient care is the generation of skilled and clinically confident neurologists. This is especially relevant with the UK-wide shortage of neurologists. ${ }^{5}$

Several key themes have emerged from this work. While some are intuitive, many of our findings highlight the value of particular structures or organisational features within departments that transcend technical aspects of training, such as formal teaching. These relate primarily to the interaction between the trainee and his/her supervisor, the differing needs of trainees according to their level of experience and the structure and culture in which training is embedded in a given department. While some variation in training is determined by the need to deliver local clinical services, we suspect that other factors, such as attitudes, practices and policies within individual institutions, also play a role. Trainee satisfaction, including well-being, is a topical issue, with increasing concern about retention; we identified wider (deanery-associated) issues of long commutes and short notice about rotations that often exacerbated dissatisfaction with training experience.

Box 2 summarises our key recommendations. We recognise that these may not be applicable to all training environments, and also that we have not addressed several important aspects of training such as rota design and access to, and integration of, research training opportunities. There are some additional limitations to our study:

First, sites with fewer than three trainees were excluded from the GMC National Training Survey data and therefore from our study. There are likely to be specific opportunities and challenges with training in smaller sites that we have not captured, but some of our themes are likely to be relevant.

Second, although there is an assumption that GMC National Training Survey results reliably inform us about the quality of training in the UK, there is no published evidence demonstrating their correlation with training outcomes. We therefore compared overall satisfaction scores with the latest available data on outcomes at annual reviews in neurology; 
- Case study 1. At one top-performing site, the neurologist supervising referrals rotates weekly and the neurologist who supervises inpatient care rotates monthly. Neurologists switch their routine outpatient clinics to rapid access clinics during the week they supervise referrals. In parallel, trainees cover referrals for several half-day sessions per week and meet the supervising neurologist at a designated time to discuss cases and review inpatients. The trainee covering referrals in the morning would hand over to the next trainee ensuring adequate time to attend an afternoon clinic. Trainees at this site reported that they had 'never felt unsupervised during their training', and with reference to discussions with the supervising neurologists, 'valued these interactions as training opportunities'.

- Case study 2. At one top-performing site, new trainees start on a ward-based rotation, with the most direct supervision. Clinics are structured according to the trainee's seniority: junior trainees have fewer patients per clinic and sometimes have new and follow-up patients in separate clinics. The neurologist supervising clinics identifies specific cases of interest for junior trainees and might provide guidance on how to approach difficult cases before the clinic. Senior trainees are encouraged to take more initiative but the supervising neurologist is always available to provide advice, if needed.

- Case study 3. At one top-performing site, training rotations are firm based covering different major subspecialties for 4 month periods. Each rotation is completed twice over the training programme and includes at least one general and subspecialty clinic. There are separate rotations based at district general hospitals but training is based at one site. Rotations are discussed with the senior trainee and then agreed with the training lead prior to allocations and according to needs of each trainee. A 4-month elective period is scheduled for the penultimate or final year. This is completely flexible and provides trainees with the opportunity to gain or improve key competencies prior to completion of training.

- Case study 4. At one top-performing site, a form of teaching or dedicated educational activity is available on almost every weekday. This includes separate bedside and small-group seminar-based teaching, a neurology journal club, neurophysiology training, a departmental meeting for case-based discussions (ie, grand rounds) and external speakers, and a neuroradiology meeting that incorporates teaching for trainees. Additional informal sessions for case-based discussions with the clinical lead and peer-led sessions were also arranged. those deaneries where at least one trainee had an unsatisfactory outcome had lower overall satisfaction scores than those where all trainees had satisfactory outcomes (80.5 vs $86.6, \mathrm{p}=0.018)$. We also assessed whether year-by-year comparisons showed consistent patterns of performance; scores did not significantly differ between 2018 and each of the 5 preceding years. Irrespective of whether the national survey results reliably and consistently inform us about the quality of training or not, our focus in this paper was to describe a range of different practices, some of which may not be widely recognised, and to allow neurologists to decide how they can be used to improve training locally.

With ever-increasing demand for neurological care, and the prominence of stroke in neurological services, there is growing tension between service delivery and clinical training in the UK. A broader appreciation of effective, efficient and safe ways to train neurologists may allow trainees and trainers alike to refine their approach to training and better shape the next generation of neurologists.

Acknowledgements We are grateful to trainees and trainers at all sites who shared their experiences with insight and candour and to other members of the Association of British Neurology $(\mathrm{ABN})$ Council and Specialist Advisory Committee (SAC) for Neurology for their support.

Contributors The idea for this project was conceived by SS in collaboration with SKA and AZ. A proposal was presented to members of the ABN Council, including CJM and TTW, and the Neurology SAC, including TATH and ACP. All authors then commented on the project design. The ABN and SAC were therefore involved in the delivery of this project at an early stage. SS, AZ and SKA conducted the interviews and thematic analysis. CJM reviewed the data analysis. SS, AZ and SKA drafted the manuscript. All authors commented on the manuscript. ACP, TTW and CJM revised the manuscript. The $A B N$ Executive endorsed the paper on behalf of the ABN.

Funding The Association of British Neurology (ABN) provided financial support for travel expenses.

Competing interests None declared.

Patient consent for publication Not required.

Provenance and peer review Commissioned. Externally peer reviewed by Rhys Thomas, Newcastle-upon-Tyne, UK.

\section{REFERENCES}

1 Health Education England. Resource bank, 2019. Available: https://specialtytraining.hee.nhs.uk/Resources-Bank [Accessed Mar 2019].

2 JRCPTB. Neurology, 2019. Available: https://www.jrcptb.org. uk/specialties/neurology [Accessed March 2019].

3 General Medical Council. National training surveys reports, 2019. Available: https://www.gmc-uk.org/about/what-we-doand-why/data-and-research/national-training-surveys-reports [Accessed Mar 2019].

4 Brashear A, Vickrey BG. Burnout in neurology: extinguishing the embers and rekindling the joy in practice. Neurology 2018;91:907-8.

5 ABN. Acute neurology survey: ABN March 2017, 2019. Available: https://www.theabn.org/resources/abn/a/abn-acuteneurology-survey-2017.html [Accessed Mar 2019]. 